\title{
PQQ ameliorates skeletal muscle atrophy, mitophagy and fiber type transition induced by denervation via inhibition of the inflammatory signaling pathways
}

\author{
Wenjing Ma", Ru Zhang ${ }^{2 \#}$, Ziwei Huang ${ }^{1}$, Qiuyu Zhang ${ }^{1}$, Xiaoying Xie ${ }^{1}$, Xiaoming Yang ${ }^{1}$, Qi Zhang ${ }^{1}$, \\ Hua Liu ${ }^{3}$, Fei Ding ${ }^{1}$, Jianwei Zhu ${ }^{4}$, Hualin Sun ${ }^{1}$
}

${ }^{1}$ Key Laboratory of Neuroregeneration of Jiangsu and Ministry of Education, Jiangsu Clinical Medicine Center of Tissue Engineering and Nerve Injury Repair, Co-Innovation Center of Neuroregeneration, Nantong University, Nantong 226001, China; ${ }^{2}$ Department of Imaging, The Second Affiliated Hospital of Nantong University, Nantong University, Nantong 226001, China; ${ }^{3}$ Department of Orthopedics, Haian Hospital of Traditional Chinese Medicine, Haian 226600, China; ${ }^{4}$ Department of Orthopedics, Affiliated Hospital of Nantong University, Nantong 226001, China Contributions: (I) Conception and design: H Sun; (II) Administrative support: H Sun, J Zhu; (III) Provision of study materials or patients: W Ma, R Zhang, Z Huang, Q Zhang, X Xie, H Liu; (IV) Collection and assembly of data: W Ma, R Zhang, Z Huang, Q Zhang, X Xie, Q Zhang, H Liu, J Zhu; (V) Data analysis and interpretation: W Ma, R Zhang, Z Huang, X Yang, J Zhu; (VI) Manuscript writing: All authors; (VII) Final approval of manuscript: All authors.

\#These authors contributed equally to this work.

Correspondence to: Hualin Sun. Key Laboratory of Neuroregeneration of Jiangsu and Ministry of Education, Jiangsu Clinical Medicine Center of Tissue Engineering and Nerve Injury Repair, Co-Innovation Center of Neuroregeneration, Nantong University, 19 Qixiu Road, Nantong 226001, China. Email: sunhl@ntu.edu.cn; Jianwei Zhu. Department of Orthopedics, Affiliated Hospital of Nantong University, Nantong 226001, China. Email: zhujianwei_nt@163.com.

Background: Skeletal muscle atrophy involves and requires widespread changes in skeletal muscle gene expression and signaling pathway, resulting in excessive loss of muscle mass and strength, which is associated with poor prognosis and the decline of life quality in several diseases. However, the treatment of skeletal muscle atrophy remains an unresolved challenge to this day. The aim of the present study was to investigate the influence of pyrroloquinoline quinone (PQQ), a redox-active o-quinone found in various foods and mammalian tissues, on skeletal muscle atrophy, and to explore the underlying molecular mechanism.

Methods: After denervation, mice were injected intraperitoneally with saline plus PQQ (5 mg/kg/d) or saline only for 14 days. The level of inflammatory cytokines in tibialis anterior (TA) muscles was determined by quantitative real-time polymerase chain reaction (qRT-PCR) and enzyme-linked immunosorbent assay (ELISA), and the level of signaling proteins of Janus kinase 2/signal transduction and activator of transcription 3 (Jak2/STAT3), TGF- $\beta 1 / S m a d 3, J N K / p 38$ MAPK, and nuclear factor $\kappa B(N F-\kappa B)$ signaling pathway were detected by Western blot. The skeletal muscle atrophy was evaluated by muscle wet weight ratio and cross-sectional areas (CSAs) of myofibers. The mitophagy was observed through transmission electron microscopy (TEM) analysis, and muscle fiber type transition was analyzed through fast myosin skeletal heavy chain antibody staining.

Results: The proinflammatory cytokines IL-6, IL-1 $\beta$ and TNF- $\alpha$ were largely induced in TA muscles after sciatic nerve transection. PQQ can significantly reverse this phenomenon, as evidenced by the decreased levels of proinflammatory cytokines IL-6, IL-1 $\beta$ and TNF- $\alpha$. Moreover, PQQ could significantly attenuate the signal activation of Jak2/STAT3, TGF- $\beta 1 / \mathrm{Smad} 3$, JNK/p38 MAPK, and NF- $\mathrm{BB}$ in skeletal muscles after sciatic nerve transection. Furthermore, PQQ alleviated skeletal muscle atrophy, mitigated mitophagy and inhibited slow-to-fast muscle fiber type transition.

Conclusions: These results suggested that PQQ could attenuate denervation-induced skeletal muscle atrophy, mitophagy and fiber type transition through suppressing the Jak2/STAT3, TGF- $\beta 1 / \mathrm{Smad} 3$, JNK/ p38 MAPK, and NF- $\kappa \mathrm{B}$ signaling pathways. 
Keywords: Denervation; muscle atrophy; pyrroloquinoline quinone (PQQ); inflammation

Submitted Aug 11, 2019. Accepted for publication Aug 19, 2019.

doi: 10.21037/atm.2019.08.101

View this article at: http://dx.doi.org/10.21037/atm.2019.08.101

\section{Introduction}

Skeletal muscle is one of the largest and most vital organs in the body, and a plastic organ maintained by multiple pathways regulating cell and protein turnover, and also a secretory organ secreting a variety of compounds including myokines, metabolites, microRNAs, and exosomes so on $(1,2)$. There are a variety of pathophysiologic conditions, such as denervation, ageing, immobilization, sepsis and cancer, inducing skeletal muscle atrophy (3). Skeletal muscle atrophy occurs when the rates of protein degradation exceed the rates of protein synthesis, and involves in multiple distinct signaling pathways (4), and it is also associated with poor prognosis and the decline of life quality. However, the treatment of skeletal muscle atrophy remains an unresolved challenge to this day (5). Therefore, improving our understanding of the mechanisms responsible for skeletal muscle atrophy is very important to develop new clinical therapeutic strategies to prevent skeletal muscle atrophy.

Skeletal muscle atrophy characterized by loss of skeletal muscle mass, mitochondrial dysfunction, muscle fiber type transition, and a reduced physical functional capacity. Increasing evidence showed that inflammation was an important contributor and driver to skeletal muscle atrophy $(6,7)$. Systemic inflammation could promote muscle atrophy via increased ubiquitin-proteasome system (UPS) and the autophagy-lysosome system (ALS) mediated protein degradation (8). Hindlimb immobilization model suggested the presence of an early inflammatory process, as evidenced by elevated IL- 6 , IL- $1 \beta$ and TNF- $\alpha$, in skeletal muscles (9), and the proinflammatory cytokines (IL- 6, IL- $1 \beta$ and TNF- $\alpha$ ) could induce muscle proteolysis (10). IL-6 infusion resulted in muscle atrophy, and inhibition of IL-6 receptor reduced MuRF1 expression and ameliorated tail suspensioninduced skeletal muscle atrophy (4,11-13). Inhibition of IL-1 $\beta$ could prevent inflammation-induced muscle atrophy in critically ill patients $(14,15)$. TNF- $\alpha$ could induce C2C12 myotube atrophy through contributing to both hyperexpression of MAFbx and MuRF1 (16,17). Moreover, these inflammatory factors are involved in many signaling pathways, and these pathways collaborate together to trigger muscle atrophy.
Emerging evidence supports a key role for the Janus kinase/signal transduction and activator of transcription (Jak/STAT) signaling pathway in inflammation, and the Jak/ STAT pathway is now recognized as a trigger of muscle protein loss $(18,19)$. Silva et al. found that the activation of Jak/STAT3 pathway was significantly induced in muscle in mice with cachexia, and the inhibition of p-STAT3 in muscle decreased the expression of MAFbx and myostatin, alleviated muscle atrophy, and increased body weight and grip strength (19). Smith et al. demonstrated that controlled mechanical ventilation (CMV)-induced diaphragm atrophy accompanied with marked increases in STAT3 phosphorylation. Inhibition of Jak signaling could prevent the activation of STAT3 during CMV, reverse the pathologic alterations in the diaphragm, and maintain normal diaphragm contractility (20). Jak2/STAT3 pathway was also activated in muscles of immobilized intensive care unit (ICU) patients, and Jak2/STAT3 inhibitors could effectively alleviate muscle atrophy, and may provide an attractive pharmacological intervention strategy in immobilized ICU patients (21). However, the role of Jak2/STAT3 pathway in denervated muscle atrophy remains unclear.

Nuclear factor $\kappa \mathrm{B}(\mathrm{NF}-\kappa \mathrm{B})$ is best known for its proinflammatory action, and emerging evidence suggests that the inflammation-sensitive NF- $\kappa B$ pathway may contribute to muscle atrophy $(8,22)$. NF- $\kappa \mathrm{B}$ activation (phospho-p65) is required for $\mathrm{C} 2 \mathrm{C} 12$ myotube atrophy induced exogenous TNF- $\alpha$, IL- $1 \alpha$ or IL-1 $\beta$, and inhibition of $\mathrm{p} 65$ could reverse C2C12 myotube atrophy due to TNF- $\alpha$, IL- $1 \alpha$ or IL-1 $\beta$ (23). $\mathrm{NF}-\kappa \mathrm{B}$ activation (phospho-p65) was markedly induced in muscle in mouse with food deprivation, and was critical for the subsequent muscle atrophy. While, NF- $\mathrm{\kappa B}$ inhibition in muscle could prevent muscle atrophy induced by fasting (24). Cachectic factors TNF- $\alpha$ could activate NF- $\kappa \mathrm{B}$ transcription pathway and p38 MAPK pathway, inducing skeletal muscle atrophy via upregulating MuRF1 $(25,26)$, and p38 MAPK pathway is a trigger of the upregulation of MuRF1 (25). The activation of p38 MAPK and JNK were markedly induced by cigarette smoke extract in myotubes, and the p38 MAPK inhibitor significantly reduced USP-19 gene expression and alleviated muscle atrophy (27). Schellino et al. demonstrated 
that the activation of JNK in spinal muscular atrophy (SMA) mouse model was remarkably induced, and JNKinhibitor could improve the trophism of SMA muscular fibers and motor performances (28). The inhibition of the JNK signaling pathway could also prevent lipopolysaccharide (LPS)-mediated Atrogin-1/MAFbx expression in C2C12 myotubes (29). Additionally, p38 MAPK and JNK were also activated, and augmented the expression of MAFbx in skeletal muscles suffered from limb immobilization and denervation $(30,31)$. However, the exact molecular mechanisms still need to be further explored.

TGF- $\beta 1$ is a multifunctional cytokine involving in the activation of satellite cells, the formation of connective tissue and the regulation of immune response intensity (32). In skeletal muscle, TGF- $\beta 1$ could induce skeletal muscle atrophy, requiring Smad3 and JNK activation, and inhibition of Smad3 and JNK1/2 activation could reverse the atrophic effect of TGF- $\beta(33,34)$. TGF- $\beta 1$ and $p-S m a d 3$ showed significantly increase in murine model of amyotrophic lateral sclerosis, and targeting TGF- $\beta$ signaling pathway might be a therapeutic approach to improve muscle function in several degenerative diseases (35). TGF- $\beta 1$ was also induced in denervated muscle and promoted skeletal muscle atrophy (36). Smad3 was an important downstream signaling molecule for TGF- $\beta$, and Smad 3 was significantly activated in hindlimb unloading (HU)-induced muscle atrophy (37). Other study showed that inhibition of Smad3 could suppress STAT3 signaling (38).

In summary, these signaling pathways, such as Jak2/ STAT3, TGF- $\beta 1 /$ Smad3, JNK/p38 MAPK and NF- $\kappa$ B signaling pathways, are all involved in muscle atrophy, and there are interactions or networks between these pathways to regulate muscle atrophy, which makes the process of muscle atrophy very complicated. Thus, if we only intervene in one pathway to protect muscle atrophy, we may not achieve the satisfactory effect.

Pyrroloquinoline quinone (PQQ), an aromatic tricyclic o-quinone, becomes an attractive bio-agent due to its multifunction, including anti-inflammatory property $(39,40)$. Liu et al. found that PQQ could reduce the production of proinflammatory cytokines (TNF- $\alpha$ and IL-6), and inhibit the phosphorylation level of p65, p38 and JNK in IL-1 $\beta$-stimulated human synovial sarcoma SW982 cells (40). PQQ could inhibit the activation of TGF- $\beta 1 /$ Smad signaling in Bmi-1 knocked down human renal proximal tubular epithelial (HK2) cells (41). As reported above, PQQ can inhibit proinflammatory cytokines and their downstream signaling pathways. However, it is not clear whether PQQ could protect against denervated skeletal muscle atrophy through alleviating inflammation. Hence, the aim of current study is to investigate the influence of PQQ on skeletal muscle atrophy, and to explore the underlying molecular mechanism.

\section{Methods}

\section{Animal treatment}

All animal procedures in this study were carried out in accordance with the recommendations of the Institutional Animal Care and Use Committee of Nantong University, and were approved by the Institutional Animal Care and Use Committee of Nantong University. Animals were subjected to unilateral sciatic nerve transection as described previously (42), followed by intraperitoneal injection of saline $(100 \mu \mathrm{L})$ (Den group), or PQQ $(5 \mathrm{mg} / \mathrm{kg} /$ day $)$ in $100 \mu \mathrm{L}$ saline (PQQ group) every day. The choice of PQQ concentration is based on our previous study (42). Intraperitoneal injection of $100 \mu \mathrm{L}$ saline was administered to the animals received shamoperation (Ctrl group). After 14 days, mice were anesthetized and muscle tissues were obtained, weighed, and frozen in liquid nitrogen for further investigation.

\section{Quantitative real-time polymerase chain reaction ( $q R T$ - PCR)}

Total RNA was extracted from muscles with TRIZOL reagent (Invitrogen, Carlsbad, CA, USA), subsequently the cDNA was synthesized. The qRT-PCR was performed with the iTaq Fast SYBR Green Supermix (Bio-Rad, Hercules, CA, USA) under the manufacturer's instructions. Quantitative data were acquired and analyzed under the Applied Biosystems 7500 RT-PCR system (Applied Biosystems, Foster City, CA, USA). The RT-PCR conditions were as follows: $42{ }^{\circ} \mathrm{C}$ for $20 \mathrm{~min}$ and then 40 cycles at $95^{\circ} \mathrm{C}$ for $5 \mathrm{~min}, 94^{\circ} \mathrm{C}$ for $20 \mathrm{~s}$, and $72{ }^{\circ} \mathrm{C}$ for $42 \mathrm{~s}$ (4). The primers were as follows: mouse $I L-6$ F: CTGCAAGAGACTTCCATCCAG, R: AGTGGTATAGACAGGTCTGTTGG; mouse $I L-1 \beta$ F: GAAATGCCACCTTTTGACAGTG, R: TGGATGCTCTCATCAGGACAG; mouse $T N F-\alpha$ F: CAGGCGGTGCCTATGTCTC, R: CGATCACCCCGAAGTTCAGTAG; mouse $\beta$-actin F: GCTTCTAGGCGGACTGTTACT, R: GCCTTCACCGTTCCAGTTTTT. The relative mRNA expression was measured through the $2^{-\Delta \Delta \mathrm{Ct}}$ method (43). 


\section{Western blot analysis}

Western blot analysis was used to determine the protein expression as described previously (4). The total proteins were extracted from muscles with RIPA Lysis Buffer, after centrifugation, soluble proteins were quantified with BCA Kit (Beyotime, Nantong, China) and separated on sodium dodecyl sulfate polyacrylamide gel electrophoresis (SDS-PAGE). Subsequently, the proteins in the gel were transferred to polyvinylidene difluoride (PVDF) membranes (Millipore Corp, Billerica, MA, USA). The PVDF membranes were blocked with $5 \%$ nonfat dry milk in Trisbuffer saline, then the PVDF membranes were incubated with primary antibodies: mouse anti-MHC polyclonal antibody (R\&D Systems, Minneapolis, MN, USA), rabbit anti-MuRF1, MAFbx (Fbx32), LC3B, PINK1, BNIP3, ATG7, TGF- 31 , Smad3, p-Smad3, p65, p-p65, p38, p-p38, p-JNK, JNK, PGC-1 $\alpha$, Sirt1 and beta tubulin antibodies (Abcam, Cambridge, UK), Jak, STAT3 and p-STAT3 (CST, USA), Troponin I-FS and Troponin I-SS (Santa Cruz, USA), at $4{ }^{\circ} \mathrm{C}$ overnight. Then, the PVDF membranes were incubated with horseradish peroxidase (HRP) conjugated corresponding $\operatorname{IgG}$ antibodies for $2 \mathrm{~h}$. The target proteins on the PVDF membranes were visualized by enhanced chemiluminescence (Thermo Scientific, Park Ellisville, MO, USA), and the intensity values were obtained for further normalization against loading control.

\section{Muscle fiber cross-sectional area (CSA) size}

The fiber CSA of skeletal muscles was determined by laminin staining. Briefly, muscles were fixed, flash-frozen, and sectioned on cryostat with $10 \mu \mathrm{m}$ thickness. The sections were incubated with anti-laminin antibody (1:200) (Abcam, Cambridge, UK) at $4{ }^{\circ} \mathrm{C}$ overnight. The following day, sections were washed and incubated with the Alexa Fluor secondary antibody (1:400) (Invitrogen Antibodies, Waltham, MA, USA) for $30 \mathrm{~min}$ at room temperature. Then, the sections were imaged by fluorescence microscopy (Zeiss, Germany) and the CSA of myofibers was obtained with ImageJ software (NIH, Bethesda, MD, USA).

\section{Muscle fiber type transition analysis}

The fiber type transition is observed in the soleus muscles due to that the change of soleus muscle fiber type was significant after denervation (44). The sample processing method was similar to the CSA analysis. Tissue sections were incubated with Anti-Fast Myosin Skeletal Heavy chain antibody (1:200) (Abcam, Cambridge, UK) for 12 hours at $4{ }^{\circ} \mathrm{C}$. Subsequently, the sections washed and incubated with the Alexa Fluor secondary antibody (1:400) (Invitrogen Antibodies, Waltham, MA, USA) for $30 \mathrm{~min}$ at room temperature. Then, the sections were imaged by fluorescence microscopy (Zeiss, Germany).

\section{Enzyme-linked immunosorbent assay (ELISA)}

The content of IL-1 $\beta$, IL- 6 and TNF- $\alpha$ was measured using appropriate commercial ELISA analysis (4). Briefly, ELISA plates (Beyotime, Haimen, China) were washed and incubated with $100 \mu \mathrm{L}$ muscle lysates from the TA muscles at $37{ }^{\circ} \mathrm{C}$ for $2 \mathrm{~h}$. Following, ELISA plates were washed and incubated with biotinylated polyclonal antiIL-1 $\beta$, anti-IL- 6 or anti-TNF- $\alpha$ antibodies at $37{ }^{\circ} \mathrm{C}$ for $1 \mathrm{~h}$. Subsequently, ELISA plates were washed and incubated with HRP-Streptavidin at $37^{\circ} \mathrm{C}$ for $20 \mathrm{~min}$ in dark. Finally, enzyme activity was read at $450 \mathrm{~nm}$ in a microplate spectrophotometer.

\section{Transmission electron microscopy (TEM) analysis}

TEM analysis was used to observe the changes of mitochondria in skeletal muscles. The detailed procedures of TEM for muscle were previously reported (45). Briefly, muscles were first fixed in $2.5 \%$ glutaraldehyde, subsequently were fixed in $1 \%$ osmium tetroxide. Finally, muscle sections were analyzed by TEM (HT7700, Hitachi, Tokyo, Japan).

\section{Statistical analysis}

All data in this study were analyzed with one-way analysis of variance (ANOVA), followed by the Tukey's multiple comparisons test to detect differences between groups. All statistical analyses in current study were performed with GraphPad Prism software (version 7.0) (San Diego, CA, USA). $\mathrm{P}<0.05$ was regarded as statistically significant.

\section{Results}

\section{$P Q Q$ inhibited proinflammatory cytokines in denervated skeletal muscles}

After denervation, mice were injected intraperitoneally with saline vehicle plus PQQ $(5 \mathrm{mg} / \mathrm{kg} / \mathrm{d}$ ) or saline vehicle only (Den) for 14 days. After sham operation, mice were 

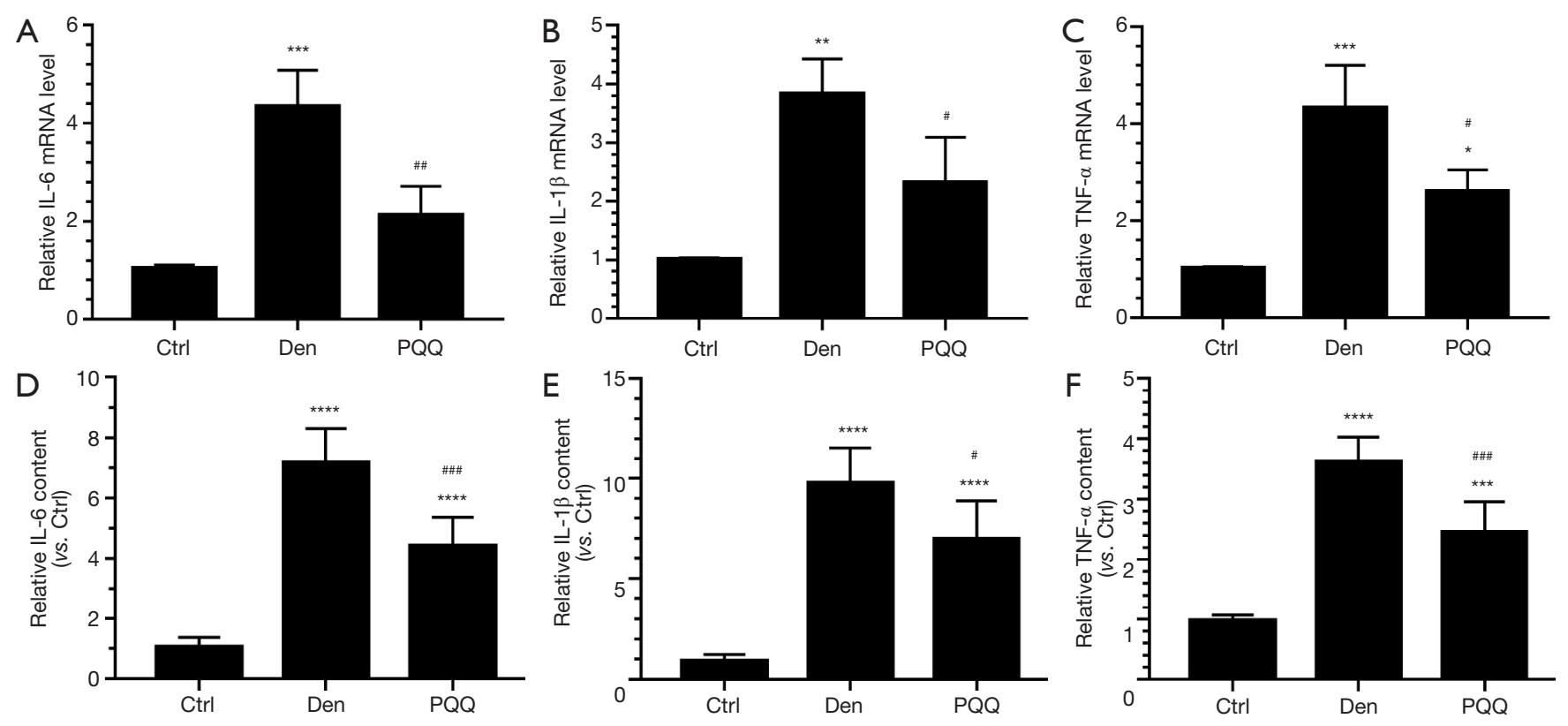

Figure 1 PQQ inhibited proinflammatory cytokines in the TA muscles suffered from sciatic nerve injury. After denervation, mice were injected intraperitoneally with saline vehicle plus PQQ (5 mg/kg/d) or saline vehicle only (Den) for 14 days. After sham operation, mice were injected intraperitoneally with saline vehicle (Ctrl) for 14 days. Then, the inflammatory cytokines were determined through ELISA and qRT-PCR analyses. (A,B,C) The qRT-PCR analyses of the expression of proinflammatory cytokines $I L-6, I L-1 \beta$ and TNF- $\alpha$ in TA muscles; (D,E,F) the relative content of proinflammatory cytokines, including IL-6, IL-1 $\beta$ and TNF- $\alpha$, were determined by ELISA. Data are expressed as mean $\pm \mathrm{SD} .{ }^{*}, \mathrm{P}<0.05 ;{ }^{* *}, \mathrm{P}<0.01 ;{ }^{* * *}, \mathrm{P}<0.001 ;{ }^{* * *}, \mathrm{P}<0.0001$ vs. Ctrl. ${ }^{*}, \mathrm{P}<0.05 ;{ }^{\# \#}, \mathrm{P}<0.01 ;{ }^{\# \#}, \mathrm{P}<0.001$ vs. Den. $\mathrm{PQQ}$, pyrroloquinoline quinone; TA, tibialis anterior; ELISA, enzyme-linked immunosorbent assay; qRT-PCR, quantitative real-time polymerase chain reaction; $\mathrm{SD}$, standard deviation.

injected intraperitoneally with saline vehicle (Ctrl) for 14 days. Then, the inflammatory cytokines were determined through ELISA and qRT-PCR analyses. The present study showed that inflammation was significantly induced in the tibialis anterior (TA) muscles after sciatic nerve transection, as evidenced by the increased proinflammatory cytokines, such as IL-6, IL-1 $\beta$ and TNF- $\alpha$ in the denervated TA muscles. Interestingly, PQQ could decrease the production of IL-6, IL- $1 \beta$ and TNF- $\alpha$ in the denervated TA muscles, as shown in Figure 1. These findings suggested that PQQ could inhibit inflammation through decreasing the proinflammatory cytokines in denervated skeletal muscles.

\section{$P Q Q$ blocked the activation of inflammatory signaling pathway in denervated skeletal muscles}

The signaling pathways involved in inflammatory factors IL-6, IL- $1 \beta$ and TNF- $\alpha$ mainly include Jak2/ STAT3, TGF- $\beta 1 / \mathrm{Smad} 3, \mathrm{JNK} / \mathrm{p} 38$ MAPK and NF- $\mathrm{KB}$ signaling pathways. In this study, we found that Jak2/STAT3,
TGF- $\beta 1 /$ Smad3, JNK/p38 MAPK and NF- $\kappa$ B signaling pathways were significantly activated in denervated TA muscles, as evidenced by the increased levels of Jak2, TGF- $\beta 1$, and phosphorylated Stat3, Smad3, JNK, p38 and p65 in the TA muscles after sciatic nerve transection, as illustrated in Figure 2, which indicated that these signaling pathways involved in inflammation were remarkably induced after denervation in skeletal muscles. Intriguingly, PQQ could decrease the levels of Jak2, TGF- $\beta 1$, and phosphorylated STAT3, Smad3, JNK, p38, p65 in the TA muscles after sciatic nerve transection, as illustrated in Figure 2, which suggested that PQQ could block the activation of inflammatory signaling pathway in denervated skeletal muscles.

\section{$P Q Q$ attenuated denervation-induced skeletal muscle atrophy}

In order to explore the effects of PQQ on muscle atrophy induced by denervation, the muscle mass and myofiber 
CSA of TA muscles were determined. In current study, we found that denervation-induced the loss of muscle mass and the decreased average myofiber CSA of TA muscles was significantly reversed by PQQ administration, which was illustrated by increased myofiber CSA and TA muscle mass, as shown in Figure 3. Additionally, we found that PQQ could inhibit denervation-induced decrease of MHC in TA muscles through suppressing the ubiquitin proteasome system proteolytic activity, as evidenced by the decreased levels of muscle-specific E3 ubiquitin ligases Atrogin-1/ MAFbx and MuRF1 in denervated skeletal muscles, as illustrated in Figure 3. These observations suggested that PQQ attenuated denervation-induced skeletal muscle atrophy through, at least in part, inhibiting ubiquitin proteasome proteolytic pathway.

\section{$P Q Q$ suppressed denervation-induced mitophagy in skeletal muscles}

Mitochondrial dysfunction is associated with skeletal muscle atrophy (46). Therefore, we investigated the effects of PQQ on autophagosomes or autophagic vacuoles and autophagy-related molecules (ATG7, PINK1, BNIP3 and LC3B) in skeletal muscle upon denervation. Our data showed that mitophagy was considerably activated in the TA muscles after sciatic nerve transection, as illustrated by the increased autophagosomes or autophagic vacuoles and the elevated autophagy-related molecules, including ATG7, PINK1, BNIP3 and LC3B (Figure 4). Interestingly, the autophagosomes or autophagic vacuoles were significantly reduced, accompanied by the decreased autophagy-related molecules, in the muscles of denervated mice treated with PQQ compared with that treated with saline (Figure 4). Collectively, these findings indicated that PQQ suppressed denervation-induced mitophagy and the expression of autophagy-related molecules in denervated skeletal muscles.

\section{$P Q Q$ suppressed slow-to-fast myofiber-type transition in skeletal muscles after denervation}

The myofiber-type transition is one of important features of skeletal muscle atrophy (47). The soleus muscles were chosen to further study in this observation due to that the change of soleus muscle fiber type was significant after denervation (44). To investigate the effects of PQQ on myofiber-type transition induced by denervation, the soleus muscles were harvested to undergo MyHC II staining analysis after sciatic nerve transection. MyHC II staining analysis showed that the percentage of $\mathrm{MyHC}$ II positive fibers was markedly increased in the soleus muscles of mice after denervation compared with sham-operated mice (Figure $5 A, B$ ), which suggested that denervation induced the slow-to-fast myofiber-type transition in soleus muscles. Interestingly, the denervation-induced slow-to-fast myofiber-type transition was rescued in soleus muscles of the mice treated with PQQ, as evidenced by the decreased percentage of MyHC II positive fibers (Figure $5 A, B$ ). Additionally, Troponin I-SS and Troponin I-FS antibodies were used to distinguish the slow and fast skeletal muscle fibers with western blotting, respectively. Our results showed that the expression of Troponin I-SS was decreased and the expression of Troponin I-FS was increased in denervated soleus muscles and PQQ could reverse the phenotype induced by denervation, which was consistent with the results from $\mathrm{MyHC}$ II staining analysis (Figure $5 C, D, E)$. PGC-1 $\alpha$ and Sirt1 are all involved in maintaining the oxidative fiber type. In current study, PGC- $1 \alpha$ and Sirt1 all displayed a significant decrease in denervated soleus muscles and this decrease of PGC- $1 \alpha$ and Sirt1 was rescued by PQQ administration (Figure $5 F, G$ ). These findings demonstrated that PQQ reversed slow-to-fast myofibertype transition through, at least in part, enhancing the expression of PGC-1 $\alpha$ and Sirt1 in skeletal muscles after denervation.

\section{Discussion}

Skeletal muscle atrophy, characterized by loss of muscle mass, shrinkage of muscle fibers, mitochondrial dysfunction, alteration in fiber-type composition, and reduced physical function, occurs when protein degradation exceeds protein synthesis under the conditions of denervation, cancer cachexia and aging (48). More recently, understanding about the molecular mechanisms regulating skeletal muscle atrophy has taken a quantum leap, though most of the current researches were focused on the role of various effector kinases and some downstream transcription factors $(49,50)$. The initial events that trigger the activation of atrophying signaling pathways remain poorly defined. Our study demonstrated that inflammation might be a major upstream regulator of skeletal muscle atrophy in response to denervation, as evidenced by the increased proinflammatory cytokines and the activated inflammatory signaling pathways in denervated skeletal muscles, which was in agreement with recent reports that inflammation was an important contributor and driver to skeletal muscle atrophy 

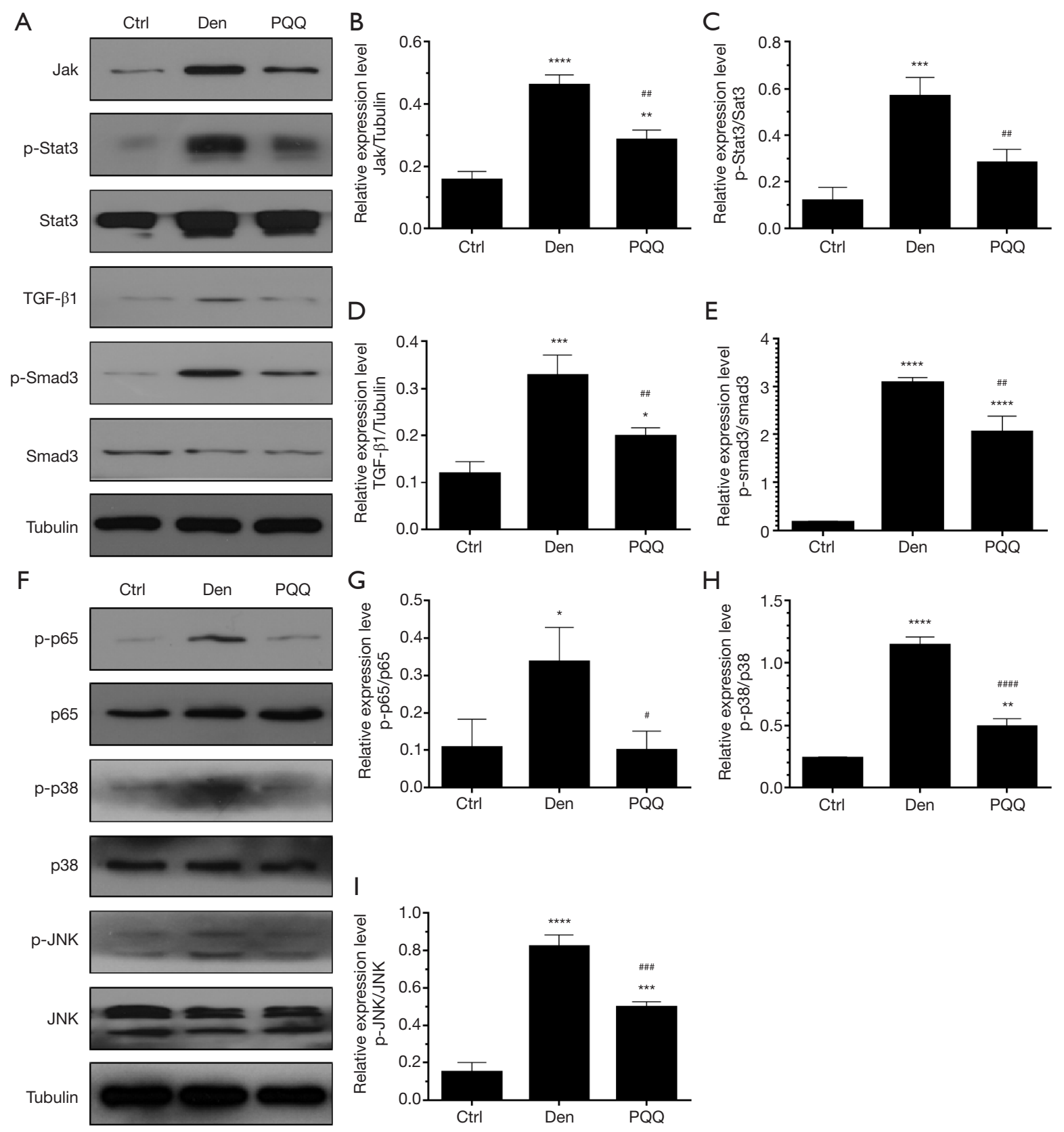

Figure 2 PQQ blocked the activation of inflammatory signaling pathway induced by sciatic nerve injury in mice TA muscles. After denervation, mice were injected intraperitoneally with saline vehicle plus PQQ ( $5 \mathrm{mg} / \mathrm{kg} / \mathrm{d}$ ) or saline vehicle only (Den) for 14 days. After sham operation, mice were injected intraperitoneally with saline vehicle (Ctrl) for 14 days. Then the TA muscles were harvested to undergo western blotting analysis. (A,F) Representative western blot images were shown for Jak, STAT3, TGF- $\beta 1$, Smad3, p65, p38 and JNK and phosphorylated STAT3, Smad3, p65, p38 and JNK; (B,C,D,E) relative expression of Jak, STAT3, TGF- $\beta 1$ and Smad3 levels in different muscle samples; $(\mathrm{G}, \mathrm{H}, \mathrm{I})$ relative expression of p65, p38 and JNK levels in different muscle samples. Data are expressed as mean \pm SD. *,

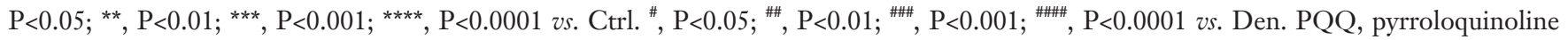
quinone; TA, tibialis anterior; Jak, Janus kinase; STAT, signal transduction and activator of transcription; SD, standard deviation. 


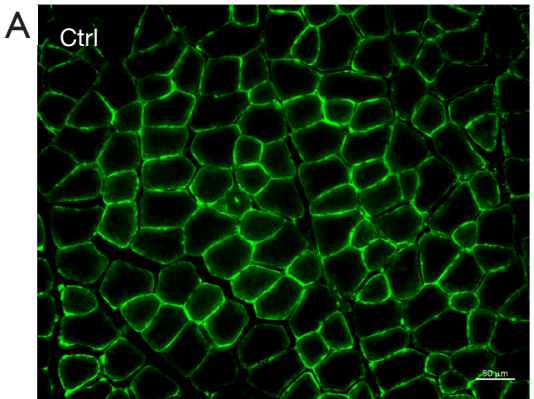

B

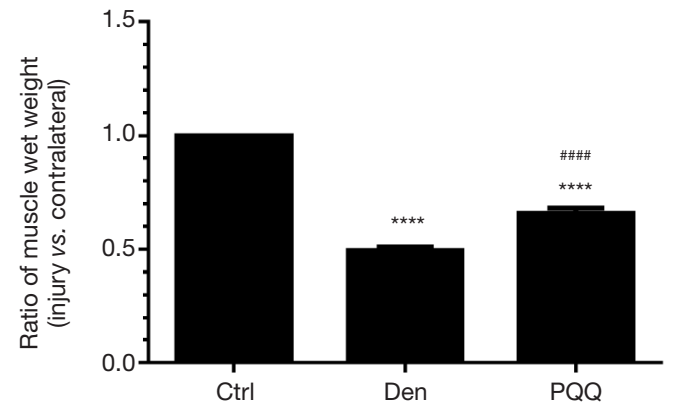

D

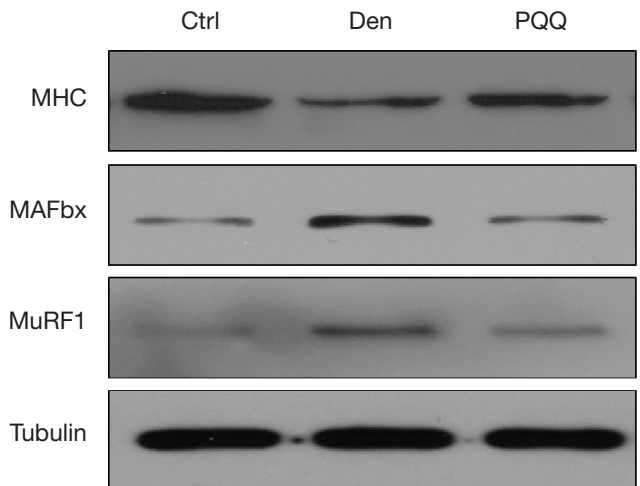

F

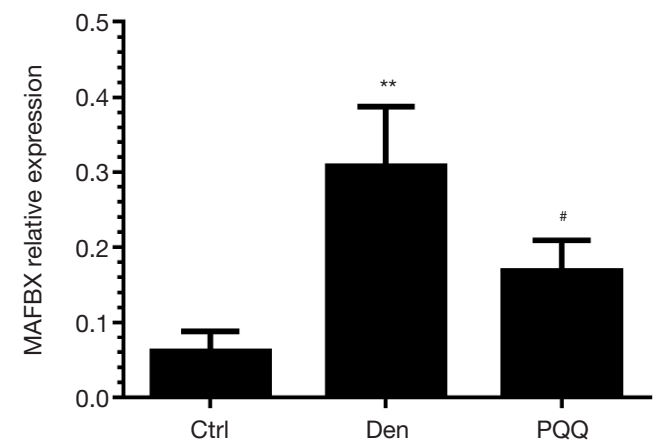

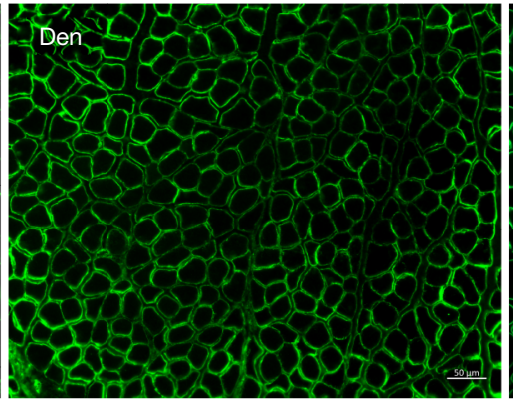

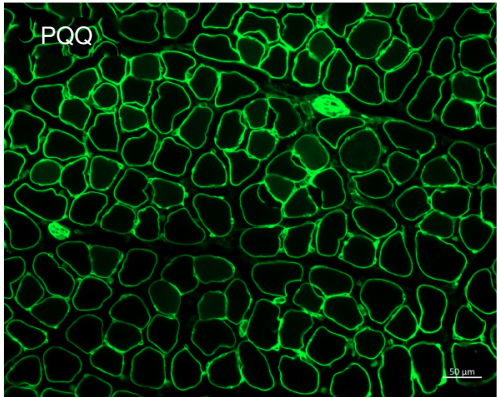

C

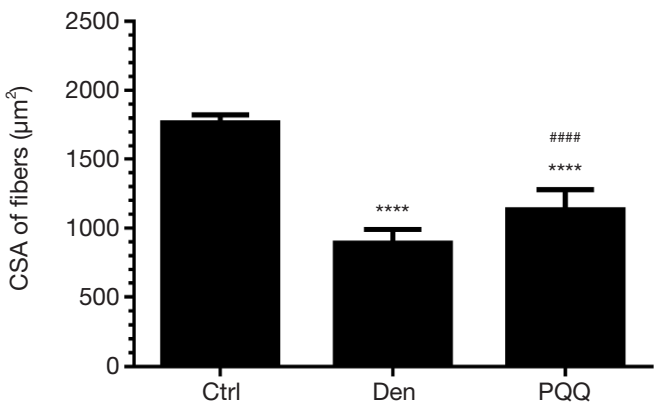

$E$

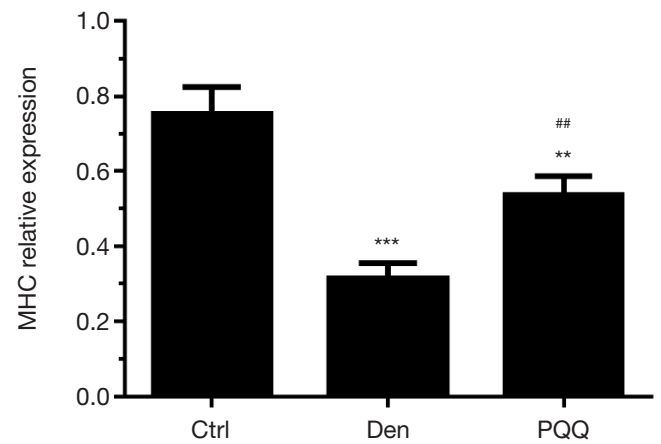

G

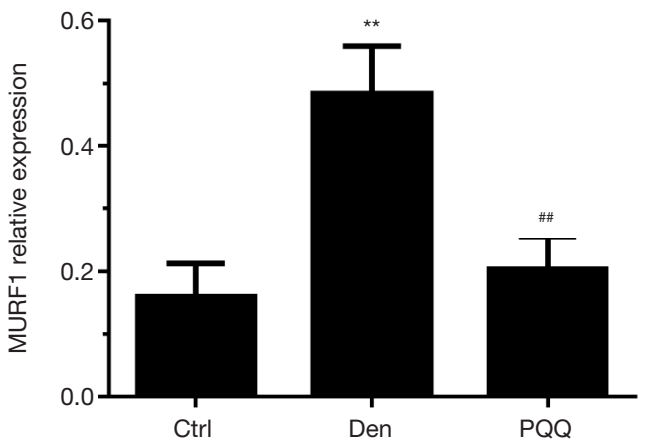

Figure 3 PQQ ameliorated denervation-induced skeletal muscle atrophy. After denervation, mice were injected intraperitoneally with saline vehicle plus PQQ (5 mg/kg/d) or saline vehicle only (Den) for 14 days. After sham operation, mice were injected intraperitoneally with saline vehicle (Ctrl) for 14 days. Then the TA muscles were harvested to undergo laminin staining analysis. (A) Representative images of laminin-stained TA muscles cross-sections. Green indicates laminin staining. Scale bar $50 \mu \mathrm{m}$; (B) wet weight ratio of TA muscles from each group; (C) mean \pm SD of CSA in TA fibers from each group; (D) representative blots of MHC, MAFbx and MuRF1 in TA muscles; (E,F,G) relative expression of MHC, MuRF1 and MAFbx in TA muscles. Data are expressed as mean $\pm \mathrm{SD}$. **, $\mathrm{P}<0.01 ;{ }^{* * *}, \mathrm{P}<0.001 ;{ }^{* * *}, \mathrm{P}<0.0001$ vs. Ctrl. ", $\mathrm{P}<0.05$; ${ }^{\# \prime}, \mathrm{P}<0.01$; \#\#\#, $\mathrm{P}<0.0001$ vs. Den. PQQ, pyrroloquinoline quinone; TA, tibialis anterior; CSA, cross-sectional area; SD, standard deviation. 


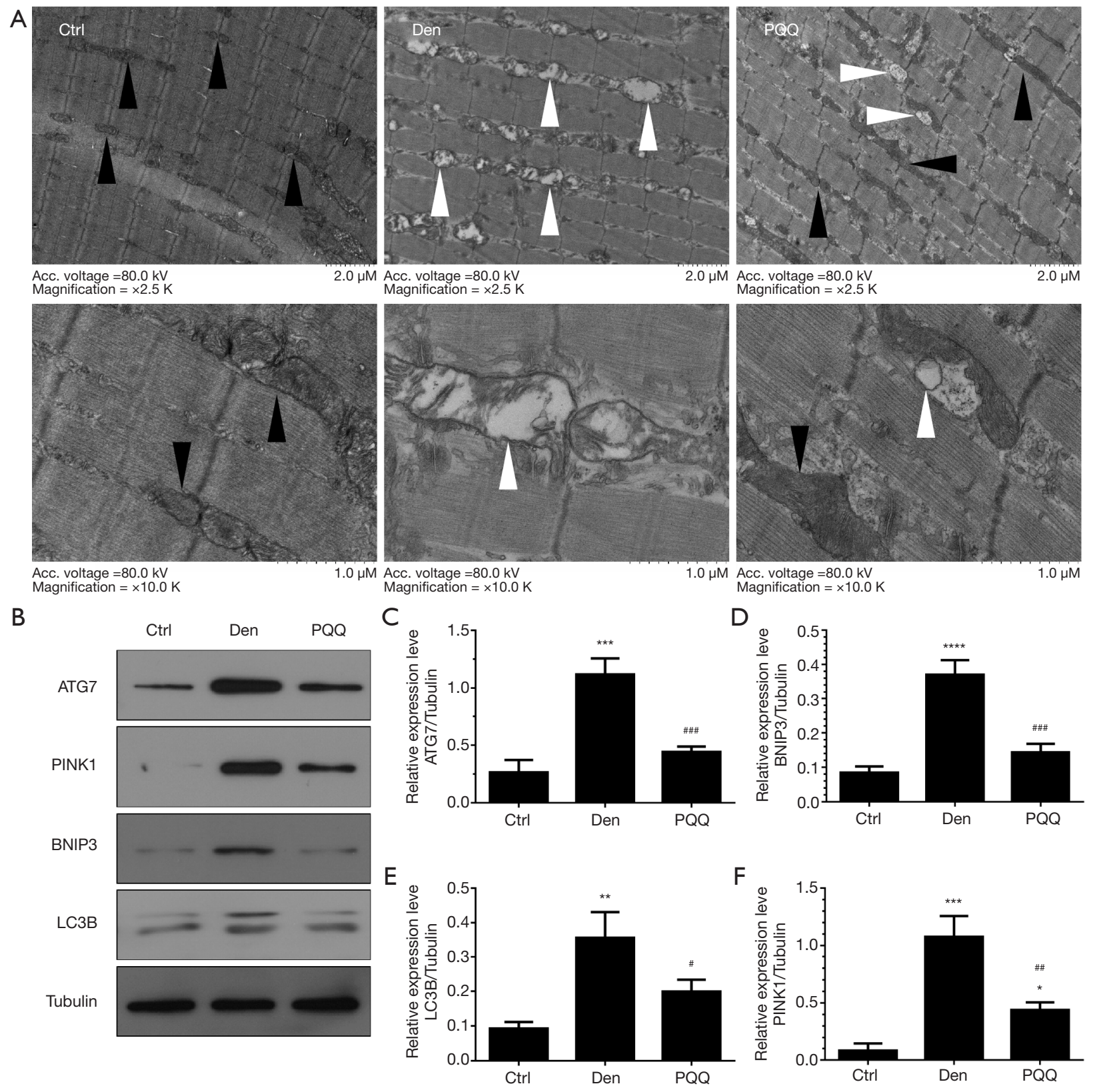

Figure 4 PQQ ameliorated mitophagy induced by sciatic nerve transection in the TA muscles. After denervation, mice were injected intraperitoneally with saline vehicle plus PQQ $(5 \mathrm{mg} / \mathrm{kg} / \mathrm{d})$ or saline vehicle only (Den) for 14 days. After sham operation, mice were injected intraperitoneally with saline vehicle (Ctrl) for 14 days. Then the TA muscles were harvested to undergo TEM analysis. (A) Representative TEM micrographs of TA muscles from different groups. The lower is a local enlargement of the upper. Black arrows indicate normal mitochondria. White arrows indicate mitophagy; (B) representative blots of autophagy genes, including ATG7, PINK1, BNIP3 and LC3B in TA muscles; (C,D,E,F) relative expression of ATG7, PINK1, BNIP3 and LC3B levels in TA muscles. Data are expressed as mean $\pm \mathrm{SD} .{ }^{*}, \mathrm{P}<0.05 ;{ }^{* *}, \mathrm{P}<0.01$; ***, $\mathrm{P}<0.001 ;{ }^{* * *}, \mathrm{P}<0.0001$ vs. Ctrl; ${ }^{*}, \mathrm{P}<0.05 ;{ }^{\# \#}, \mathrm{P}<0.01 ;{ }^{\# \# \#}, \mathrm{P}<0.001$ vs. Den. PQQ, pyrroloquinoline quinone; TA, tibialis anterior; TEM, transmission electron microscopy; SD, standard deviation. 


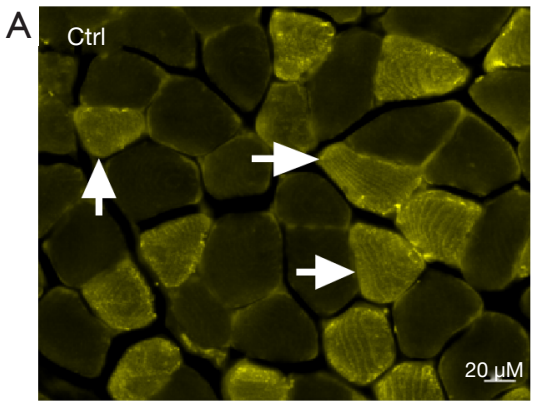

B

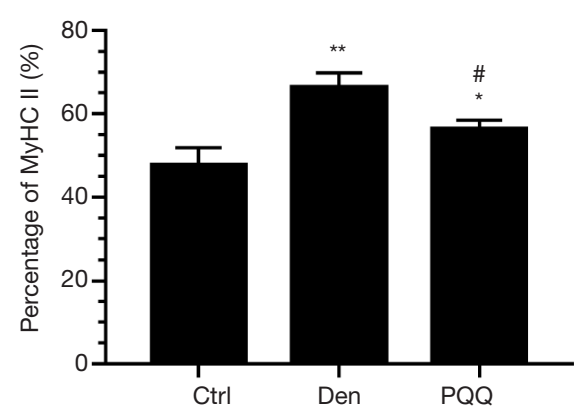

D

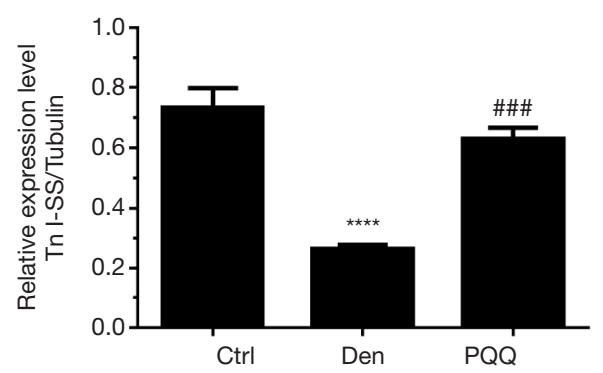

$\mathrm{F}$

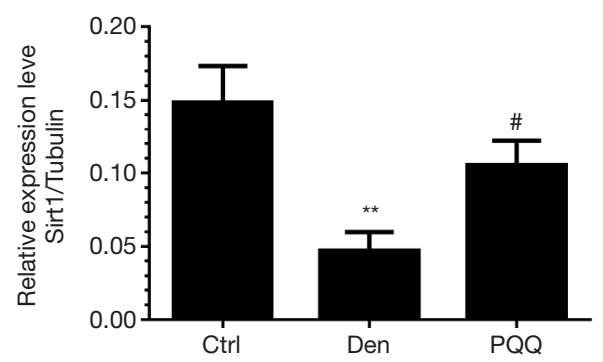

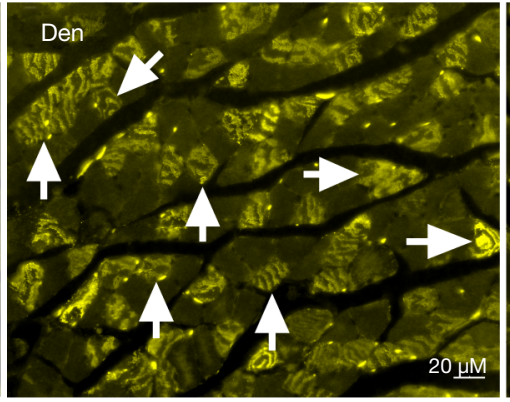

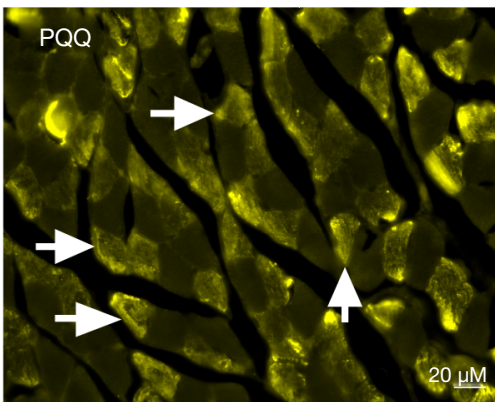

C

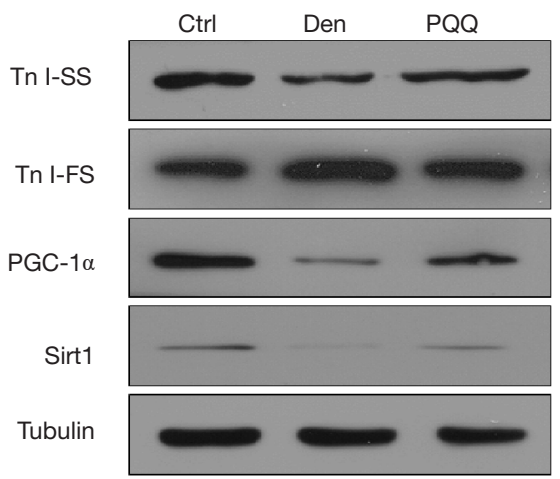

E

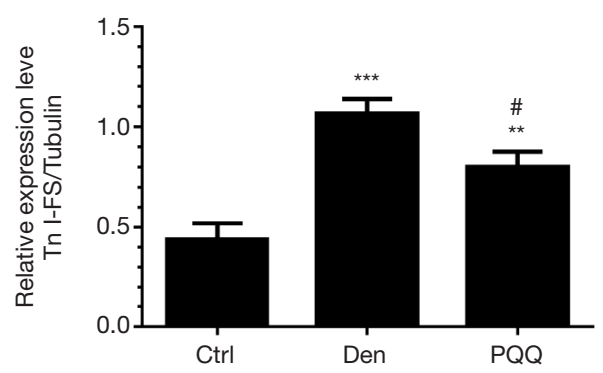

G

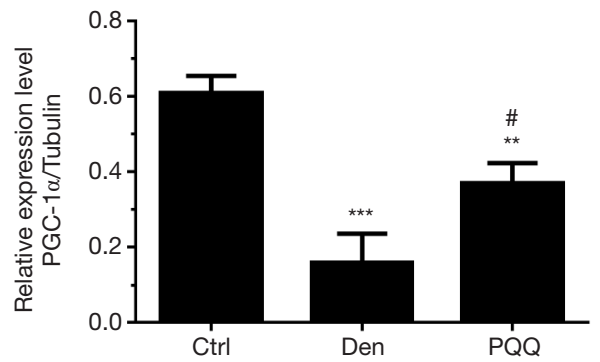

Figure 5 PQQ inhibited slow-to-fast muscle fiber type transition of soleus muscles after sciatic nerve transection. After denervation, mice were injected intraperitoneally with saline vehicle plus PQQ ( $5 \mathrm{mg} / \mathrm{kg} / \mathrm{d}$ ) or saline vehicle only (Den) for 14 days. After sham operation, mice were injected intraperitoneally with saline vehicle (Ctrl) for 14 days. Then, the soleus muscles were harvested to undergo MyHC II staining analysis. (A) Representative images of $\mathrm{MyHC}$ II-stained soleus muscles cross-sections. Kelly green indicates $\mathrm{MyHC}$ II positive staining. As shown by the white arrow in the figure. Scale bar $20 \mu \mathrm{m}$; (B) the percentage of MyHC II positive fibers; (C) representative western blot images were shown for Tn I-SS, Tn I-FS, PGC-1 $\alpha$ and Sirt1; (D,E,F,G) relative expression of Tn I-SS, Tn I-FS, PGC-1 $\alpha$ and Sirt1 levels in different muscle samples. Data are expressed as mean $\pm \mathrm{SD}$. *, $\mathrm{P}<0.05 ;{ }^{* *}, \mathrm{P}<0.01 ;{ }^{* * *}, \mathrm{P}<0.001 ;{ }^{* * * *}, \mathrm{P}<0.0001$ vs. $\mathrm{Ctrl}$;, $\mathrm{P}<0.05 ;{ }^{\# \#}, \mathrm{P}<0.001$ vs. Den. PQQ, pyrroloquinoline quinone; SD, standard deviation. 
in other conditions, such as diabetes and cancer $(6-8,51)$. Although it remains unknown what elicits the inflammatory response in atrophying muscle in response to denervation, it was of interest to note that inflammation might be an important mediator and driver to skeletal muscle atrophy, and blocking inflammation in skeletal muscle might be a novel and exciting therapeutic approach for muscle wasting in condition of denervation.

PQQ is a natural antioxidant found in soil (39). Recent studies have found that PQQ also has anti-inflammatory function. Liu et al. found that PQQ could reduce the production of TNF- $\alpha$ and IL-6, and inhibit the signal activation of $\mathrm{p} 65, \mathrm{p} 38$ and JNK in IL-1 $\beta$-stimulated human synovial sarcoma SW982 cells (40). PQQ also could inhibit the activation of TGF- $\beta 1 /$ Smad signaling (41). Although it remains unknown how PQQ suppresses inflammation, it was of interest to note that PQQ might provide a potent therapeutic benefit for the treatment of muscle atrophy.

Intriguingly, the results of the present study suggested that PQQ could alleviate skeletal muscle atrophy, mitigate mitophagy and inhibit slow-to-fast muscle fiber type transition in denervated skeletal muscles. The antimuscular atrophy function of PQQ has been confirmed by Kuo's research that demonstrated PQQ inhibiting skeletal muscle atrophy in response to denervation by activating PGC-1 $\alpha$ (52). Our previous studies have also confirmed that PQQ can alleviate muscle atrophy by inhibiting reactive oxygen species (ROS) $(42,53)$. The inflammatory cytokine could decrease PGC-1 $\alpha$ expression, and promotes ROS generation $(54,55)$. Whether PQQ improves skeletal muscle after denervation through suppressing inflammation is an area of research for future investigation.

It is not clear whether PQQ could protect against denervated skeletal muscle atrophy through alleviating inflammation. We further investigated the effects of PQQ on inflammation in skeletal muscles upon denervation. Previous studies have emphasized that inflammatory cytokines were important triggers and mediators of muscle atrophy in both animal models and patients with advanced cancer (56-59). Interestingly, our findings demonstrated that inflammation was significantly induced, and PQQ could not only reduce the production of proinflammatory cytokines IL-6, IL- $1 \beta$ and TNF- $\alpha$, but also block the signal activation of Jak2/STAT3, TGF- $\beta 1 /$ Smad3, JNK/p38 MAPK, and $\mathrm{NF}-\kappa \mathrm{B}$ in skeletal muscles after denervation. These findings revealed that PQQ could inhibit proinflammatory cytokines and their downstream signaling pathways during denervated skeletal muscles atrophy.
Jak/STAT3 pathway is now recognized as a trigger of muscle protein loss, and the inhibition of p-STAT3 in muscle decreased the expression of MAFbx and myostatin, alleviated muscle atrophy, and increased body weight and grip strength (18-21). STAT3/NF-кB inhibition increased protein synthesis in skeletal muscles in cachectic mice, alleviated muscle atrophy (60). The increased activation of STAT3 could upregulate the markers of the mitophagy/autophagy (LC3B, BNIP3) in left ventricular tissue of metastatic melanoma mice (61), which indicated that inactivation of STAT3 might inhibit mitophagy. Sirt1 suppresses the activation of STAT3, and improves the activation of PGC- $1 \alpha(62,63)$. Moreover, sirt1 overexpression could trigger the transition of myofiber fast-to-slow, increase the expression of PGC- $1 \alpha$, and decrease the expression of atrophic genes (64). In this study, PQQ suppressed the activation of Jak/STAT3, and improved the expression of Sirt1 and PGC-1 $\alpha$, accompanied with decreased the expression of UPS and ALS related proteins, reduced Troponin I-FS (fast twitch protein), and increased Troponin I-SS (slow twitch protein), which was likely responsible for the mechanism by which PQQ alleviated skeletal muscle atrophy, mitigated mitophagy and inhibited slow-to-fast muscle fiber type transition in denervated TA muscles.

$\mathrm{NF}-\kappa \mathrm{B}$ activation in skeletal muscle is important for atrophy upon denervation or cancer (24). Inflammatory signaling pathways, especially $\mathrm{NF}-\kappa \mathrm{B}$, potentially contribute to diabetes-mediated muscle atrophy (8). Overexpression of PGC- $1 \alpha$ could increase mitochondrial content (regulating myofiber-type transition), suppress the activation of NF$\kappa \mathrm{B}$, and inhibit the expression of atrophic genes such as MAFbx and MuRF1, alleviating muscle atrophy (65). In current study, PQQ could improve the expression of PGC$1 \alpha$, and suppress the activation of $\mathrm{NF}-\kappa \mathrm{B}$, which might be responsible for PQQ attenuating muscle atrophy and the transition of slow-to-fast in condition of denervation.

The activation of p38 MAPK and JNK were markedly induced by cigarette smoke extract in myotubes, and the p38 MAPK inhibitor significantly alleviated muscle atrophy (27). JNK and p38 MAPK signaling pathways were also activated, and augmented the expression of MAFbx in skeletal muscles suffered from limb immobilization and denervation (30,31). JNK/c-Jun pathway could inhibit the expression of PGC- $1 \alpha$ in cerebellar granule neurons (66). The inactivation of JNK and 338 MAPK resulted in a reduction of palmitate-induced autophagy (67). These reports revealed that inactivation of p38 MAPK and JNK might be beneficial for the treatment of muscle atrophy. Intriguingly, our findings suggested that PQQ could inhibit the activation of p38 MAPK and JNK, 


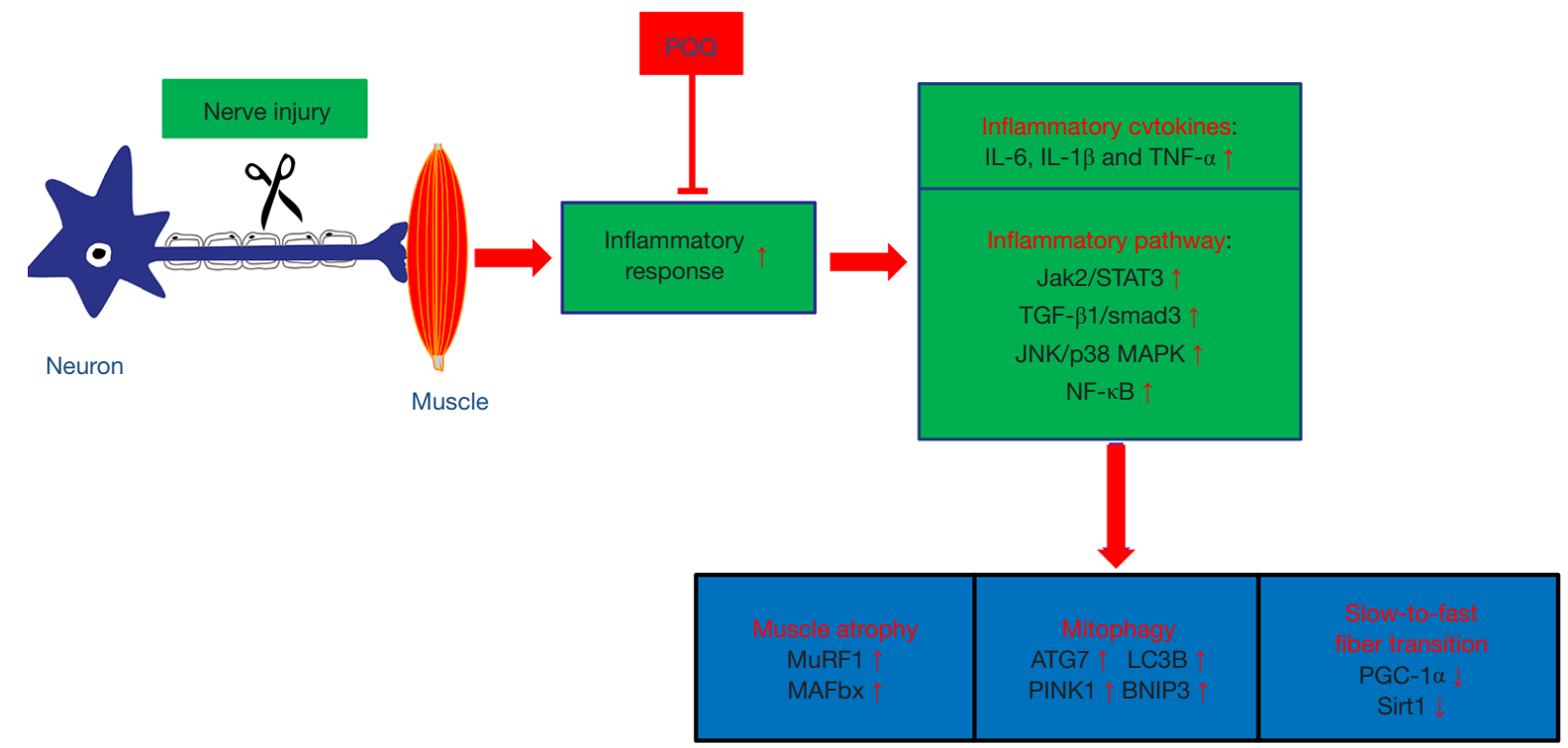

Figure 6 A schematic diagram illustrating the proposed mechanism by which PQQ attenuates skeletal muscle atrophy, mitophagy and fiber type transition on denervation. Inflammation was significantly induced in denervated skeletal muscles, as evidenced by elevated proinflammatory cytokines IL-6, IL- $\beta$ and TNF- $\alpha$, and activated Jak2/STAT3, TGF- $\beta 1 / S m a d 3$, JNK/p38 MAPK, and NF- $\kappa$ B signaling pathways in this study. Interestingly, PQQ could attenuate skeletal muscle atrophy, mitophagy and fiber type transition through suppressing the Jak2/STAT3, TGF- $\beta 1 / \mathrm{Smad} 3$, JNK/p38 MAPK, and NF- $\mathrm{BB}$ signaling pathways in denervated skeletal muscles. PQQ, pyrroloquinoline quinone; Jak2/STAT3, Janus kinase 2/signal transduction and activator of transcription 3; NF- $\kappa \mathrm{B}$, nuclear factor $\kappa \mathrm{B}$.

as evidenced by decreased levels of phosphorylated p38 MAPK and JNK in denervated TA muscles. It was likely responsible, at least part, for the mechanism by which PQQ alleviated skeletal muscle atrophy, mitigated mitophagy and inhibited slow-to-fast muscle fiber type transition induced by denervation.

TGF- $\beta 1$ could induce skeletal muscle atrophy through activating Smad3, and inactivation of $S$ mad3 could reverse the atrophic effect induced by TGF- $\beta(33,34)$. The activation of TGF- $\beta$ signaling pathway is also crucial for autophagy, and it could activate autophagy of human hepatic carcinoma HepG2 cells (68). TGF- $\beta$ / Smad3 signaling pathway could suppress the expression of PGC- $1 \alpha$ (69), and the activation of TGF- $\beta /$ Smad 3 pathway could be inhibited by sirt1 (70). These data indicated that regulating the activity of TGF- $\beta / \mathrm{Smad} 3$ pathway might be associated the skeletal muscle atrophy, autophagy and the expression of PGC-1 $\alpha$ and sirt1, which were responsible for oxidative metabolism in muscles. Thus, it can be seen that inhibition of TGF- $\beta /$ Smad3 pathway might be a novel therapeutic intervention for prevention of skeletal muscle atrophy. Interestingly, PQQ could reduce the activity of TGF- $\beta / \mathrm{Smad} 3$ pathway, as illustrated by decreased
TGF- $\beta 1$ and phosphorylated smad 3 in denervated muscles, which revealed that PQQ could alleviate skeletal muscle atrophy, mitigate mitophagy and inhibit slow-to-fast muscle fiber type transition through, at least part, inhibiting the activation of TGF- $\beta /$ Smad3 pathway.

Collectively, these findings highlighted the importance of the inflammatory signaling mechanisms in denervationinduced muscle atrophy and suggested that inflammation might be an important therapeutic target to prevent skeletal muscle wasting induced by denervation. Interestingly, PQQ could attenuate skeletal muscle atrophy, mitophagy and fiber type transition due to denervation through suppressing the Jak2/STAT3, TGF- $\beta 1 / \mathrm{Smad} 3$, JNK/p38 MAPK, and NF- $\mathrm{KB}$ signaling pathways (Figure 6). This study not only enriches the molecular regulatory mechanism of denervated muscle atrophy, but also provides a novel and exciting therapeutic approach for muscle wasting in condition of denervation.

\section{Acknowledgments}

Funding: This work was supported by National Natural Science Foundation of China (Grant No. 31730031, 81871554 
and 81671230), the 973 Program (Grant No. 2014CB542202 and 2014CB542203), a project funded by Jiangsu Provincial Key Medical Center and the Priority Academic Program Development of Jiangsu Higher Education Institutions (PAPD), and 2019 Jiangsu College Students Innovation and Entrepreneurship Training Program.

\section{Footnote}

Conflicts of Interest: The authors have no conflict of interests to declare.

Ethical Statement: The authors are accountable for all aspects of the work in ensuring that questions related to the accuracy or integrity of any part of the work are appropriately investigated and resolved. All animal procedures in this study were carried out in accordance with the recommendations of the Institutional Animal Care and Use Committee of Nantong University, and were approved by the Institutional Animal Care and Use Committee of Nantong University (No. 20170305-003).

\section{References}

1. Bonaldo P, Sandri M. Cellular and molecular mechanisms of muscle atrophy. Dis Model Mech 2013;6:25-39.

2. Barlow JP, Solomon TP. Do skeletal muscle-secreted factors influence the function of pancreatic $\beta$-cells? Am J Physiol Endocrinol Metab 2018;314:E297-307.

3. Tong T, Kim M, Park T. $\alpha$-cedrene, a newly identified ligand of MOR23, increases skeletal muscle mass and strength. Mol Nutr Food Res 2018:e1800173. [Epub ahead of print].

4. Wu C, Tang L, Ni X, et al. Salidroside attenuates denervation-induced skeletal muscle atrophy through negative regulation of pro-inflammatory cytokine. Front Physiol 2019;10:665.

5. Dutt V, Gupta S, Dabur R, et al. Skeletal muscle atrophy: potential therapeutic agents and their mechanisms of action. Pharmacol Res 2015;99:86-100.

6. Kim DS, Cha HN, Jo HJ, et al. TLR2 deficiency attenuates skeletal muscle atrophy in mice. Biochem Biophys Res Commun 2015;459:534-40.

7. Braun TP, Szumowski M, Levasseur PR, et al. Muscle atrophy in response to cytotoxic chemotherapy is dependent on intact glucocorticoid signaling in skeletal muscle. PLoS One 2014;9:e106489.

8. Perry BD, Caldow MK, Brennan-Speranza TC, et al.
Muscle atrophy in patients with Type 2 Diabetes Mellitus: roles of inflammatory pathways, physical activity and exercise. Exerc Immunol Rev 2016;22:94-109.

9. Caron AZ, Drouin G, Desrosiers J, et al. A novel hindlimb immobilization procedure for studying skeletal muscle atrophy and recovery in mouse. J Appl Physiol (1985) 2009;106:2049-59.

10. Komatsu R, Okazaki T, Ebihara S, et al. Aspiration pneumonia induces muscle atrophy in the respiratory, skeletal, and swallowing systems. J Cachexia Sarcopenia Muscle 2018;9:643-53.

11. Yakabe $M$, Ogawa $S$, Ota $H$, et al. Inhibition of interleukin-6 decreases atrogene expression and ameliorates tail suspension-induced skeletal muscle atrophy. PLoS One 2018;13:e0191318.

12. Haddad F, Zaldivar F, Cooper DM, et al. IL-6induced skeletal muscle atrophy. J Appl Physiol (1985) 2005;98:911-7.

13. Ma W, Xu T, Wang Y, et al. The role of inflammatory factors in skeletal muscle injury. Biotarget 2018;2:7.

14. Huang N, Kny M, Riediger F, et al. Deletion of Nlrp3 protects from inflammation-induced skeletal muscle atrophy. Intensive Care Med Exp 2017;5:3.

15. Takaesu G. Two types of TRAF6-dependent TAK1 activation in the IL-1 signaling pathway. Biotarget 2018;2:2.

16. Li J, Chan MC, Yu Y, et al. miR-29b contributes to multiple types of muscle atrophy. Nat Commun 2017;8:15201.

17. Wang DT, Yin Y, Yang YJ, et al. Resveratrol prevents TNF- $\alpha$-induced muscle atrophy via regulation of Akt/ mTOR/FoxO1 signaling in $\mathrm{C} 2 \mathrm{C} 12$ myotubes. Int Immunopharmacol 2014;19:206-13.

18. Banerjee S, Biehl A, Gadina M, et al. JAK-STAT signaling as a target for inflammatory and autoimmune diseases: current and future prospects. Drugs 2017;77:521-46.

19. Silva KA, Dong J, Dong Y, et al. Inhibition of Stat3 activation suppresses caspase- 3 and the ubiquitinproteasome system, leading to preservation of muscle mass in cancer cachexia. J Biol Chem 2015;290:11177-87.

20. Smith IJ, Godinez GL, Singh BK, et al. Inhibition of Janus kinase signaling during controlled mechanical ventilation prevents ventilation-induced diaphragm dysfunction. FASEB J 2014;28:2790-803.

21. Salah H, Fury W, Gromada J, et al. Muscle-specific differences in expression and phosphorylation of the Janus kinase 2/signal transducer and activator of transcription 3 following long-term mechanical ventilation and 
immobilization in rats. Acta Physiol (Oxf) 2018. doi: 10.1111/apha.12980.

22. Thoma A, Lightfoot AP. NF-kB and inflammatory cytokine signalling: role in skeletal muscle atrophy. Adv Exp Med Biol 2018;1088:267-79.

23. Yamaki T, Wu CL, Gustin M, et al. Rel A/p65 is required for cytokine-induced myotube atrophy. Am J Physiol Cell Physiol 2012;303:C135-42.

24. Lee D, Goldberg AL. Muscle wasting in fasting requires activation of NF- $\mathrm{KB}$ and inhibition of AKT/mechanistic target of rapamycin (mTOR) by the protein acetylase, GCN5. J Biol Chem 2015;290:30269-79.

25. Glass DJ. Skeletal muscle hypertrophy and atrophy signaling pathways. Int $\mathrm{J}$ Biochem Cell Biol 2005;37:1974-84.

26. Girven M, Dugdale HF, Owens DJ, et al. 1-glutamine improves skeletal muscle cell differentiation and prevents myotube atrophy after cytokine (TNF- $\alpha$ ) stress via reduced p38 MAPK signal transduction. J Cell Physiol 2016;231:2720-32.

27. Liu Q, Xu WG, Luo Y, et al. Cigarette smoke-induced skeletal muscle atrophy is associated with up-regulation of USP-19 via p38 and ERK MAPKs. J Cell Biochem 2011;112:2307-16.

28. Schellino R, Boido M, Borsello T, et al. Pharmacological c-Jun NH2-terminal kinase (JNK) pathway inhibition reduces severity of spinal muscular atrophy disease in mice. Front Mol Neurosci 2018;11:308.

29. Shiota C, Abe T, Kawai N, et al. Flavones inhibit LPS-induced Atrogin-1/MAFbx expression in mouse C2C12 skeletal myotubes. J Nutr Sci Vitaminol (Tokyo) 2015;61:188-94.

30. Childs TE, Spangenburg EE, Vyas DR, et al. Temporal alterations in protein signaling cascades during recovery from muscle atrophy. Am J Physiol Cell Physiol 2003;285:C391-8.

31. Paul PK, Gupta SK, Bhatnagar S, et al. Targeted ablation of TRAF6 inhibits skeletal muscle wasting in mice. J Cell Biol 2010;191:1395-411.

32. Delaney K, Kasprzycka P, Ciemerych MA, et al. The role of TGF- $\beta 1$ during skeletal muscle regeneration. Cell Biol Int 2017;41:706-15.

33. Akpulat U, Onbaşılar İ, Kocaefe YÇ. Tenotomy immobilization as a model to investigate skeletal muscle fibrosis (with emphasis on Secreted frizzled-related protein 2). Physiol Genomics 2016;48:397-408.

34. Ábrigo J, Campos F, Simon F, et al. TGF- $\beta$ requires the activation of canonical and non-canonical signalling pathways to induce skeletal muscle atrophy. Biol Chem 2018;399:253-64.

35. Gonzalez D, Contreras O, Rebolledo DL, et al. ALS skeletal muscle shows enhanced TGF- $\beta$ signaling, fibrosis and induction of fibro/adipogenic progenitor markers. PLoS One 2017;12:e0177649.

36. Yang X, Xue P, Liu X, et al. HMGB1/autophagy pathway mediates the atrophic effect of TGF- $\beta 1$ in denervated skeletal muscle. Cell Commun Signal 2018;16:97.

37. Wang J, Wang F, Zhang P, et al. PGC-1 $\alpha$ over-expression suppresses the skeletal muscle atrophy and myofiber-type composition during hindlimb unloading. Biosci Biotechnol Biochem 2017;81:500-13.

38. Tang H, L Kennedy C, Lee M, et al. Smad3 initiates oxidative stress and proteolysis that underlies diaphragm dysfunction during mechanical ventilation. Sci Rep 2017;7:14530.

39. Jonscher KR, Stewart MS, Alfonso-Garcia A, et al. Early PQQ supplementation has persistent long-term protective effects on developmental programming of hepatic lipotoxicity and inflammation in obese mice. FASEB J 2017;31:1434-48.

40. Liu Z, Sun C, Tao R, et al. Pyrroloquinoline quinone decelerates rheumatoid arthritis progression by inhibiting inflammatory responses and joint destruction via modulating NF- $\mathrm{KB}$ and MAPK pathways. Inflammation 2016;39:248-56.

41. Jin J, Lv X, Chen L, et al. Bmi-1 plays a critical role in protection from renal tubulointerstitial injury by maintaining redox balance. Aging Cell 2014;13:797-809.

42. Qiu J, Fang Q, Xu T, et al. Mechanistic role of reactive oxygen species and therapeutic potential of antioxidants in denervation- or fasting-induced skeletal muscle atrophy. Front Physiol 2018;9:215.

43. Livak KJ, Schmittgen TD. Analysis of relative gene expression data using real-time quantitative PCR and the 2(-Delta Delta C(T)) method. Methods 2001;25:402-8.

44. Midrio M, Danieli Betto D, Betto R, et al. Cordotomydenervation interactions on contractile and myofibrillar properties of fast and slow muscles in the rat. Exp Neurol 1988;100:216-36.

45. Wang D, Sun H, Song G, et al. Resveratrol improves muscle atrophy by modulating mitochondrial quality control in STZ-induced diabetic mice. Mol Nutr Food Res 2018;62:e1700941.

46. Theilen NT, Kunkel GH, Tyagi SC. The role of exercise and TFAM in preventing skeletal muscle atrophy. J Cell Physiol 2017;232:2348-58. 
47. Dumitru A, Radu BM, Radu M, et al. Muscle changes during atrophy. Adv Exp Med Biol 2018;1088:73-92.

48. Schiaffino S, Dyar KA, Ciciliot S, et al. Mechanisms regulating skeletal muscle growth and atrophy. FEBS J 2013;280:4294-314.

49. Adams CM, Ebert SM, Dyle MC. Role of ATF4 in skeletal muscle atrophy. Curr Opin Clin Nutr Metab Care 2017;20:164-8.

50. Reza MM, Subramaniyam N, Sim CM, et al. Irisin is a pro-myogenic factor that induces skeletal muscle hypertrophy and rescues denervation-induced atrophy. Nat Commun 2017;8:1104.

51. Fukawa T, Yan-Jiang BC, Min-Wen JC, et al. Excessive fatty acid oxidation induces muscle atrophy in cancer cachexia. Nat Med 2016;22:666-71.

52. Kuo YT, Shih PH, Kao SH, et al. Pyrroloquinoline quinone resists denervation-induced skeletal muscle atrophy by activating PGC- $1 \alpha$ and integrating mitochondrial electron transport chain complexes. PLoS One 2015;10:e0143600.

53. Xu T, Yang X, Wu C, et al. Pyrroloquinoline quinone attenuates cachexia-induced muscle atrophy via suppression of reactive oxygen species. J Thorac Dis 2018;10:2752-9.

54. Ruiz-Andres O, Suarez-Alvarez B, Sánchez-Ramos C, et al. The inflammatory cytokine TWEAK decreases PGC$1 \alpha$ expression and mitochondrial function in acute kidney injury. Kidney Int 2016;89:399-410.

55. Barth CR, Funchal GA, Luft C, et al. Carrageenaninduced inflammation promotes ROS generation and neutrophil extracellular trap formation in a mouse model of peritonitis. Eur J Immunol 2016;46:964-70.

56. Späte U, Schulze PC. Proinflammatory cytokines and skeletal muscle. Curr Opin Clin Nutr Metab Care 2004;7:265-9.

57. Li H, Malhotra S, Kumar A. Nuclear factor-kappa B signaling in skeletal muscle atrophy. J Mol Med (Berl) 2008;86:1113-26.

58. Ma JF, Sanchez BJ, Hall DT, et al. STAT3 promotes IFN $\gamma / \mathrm{TNF} \alpha$-induced muscle wasting in an NF- $\kappa \mathrm{B}$ dependent and IL-6-independent manner. EMBO Mol Med 2017;9:622-37.

59. Bonetto A, Aydogdu T, Jin X, et al. JAK/STAT3 pathway inhibition blocks skeletal muscle wasting downstream of IL-6 and in experimental cancer cachexia. Am J Physiol Endocrinol Metab 2012;303:E410-21.

60. Hardee JP, Counts BR, Gao S, et al. Inflammatory signalling regulates eccentric contraction-induced protein synthesis in cachectic skeletal muscle. J Cachexia Sarcopenia Muscle 2018;9:369-83.

61. Pietzsch S, Ricke-Hoch M, Stapel B, et al. Modulation of cardiac AKT and STAT3 signalling in preclinical cancer models and their impact on the heart. Biochim Biophys Acta Mol Cell Res 2019. [Epub ahead of print].

62. Nie Y, Erion DM, Yuan Z, et al. STAT3 inhibition of gluconeogenesis is downregulated by SirT1. Nat Cell Biol 2009;11:492-500.

63. Erion DM, Yonemitsu S, Nie Y, et al. SirT1 knockdown in liver decreases basal hepatic glucose production and increases hepatic insulin responsiveness in diabetic rats. Proc Natl Acad Sci U S A 2009;106:11288-93.

64. Chalkiadaki A, Igarashi M, Nasamu AS, et al. Musclespecific SIRT1 gain-of-function increases slow-twitch fibers and ameliorates pathophysiology in a mouse model of duchenne muscular dystrophy. PLoS Genet 2014;10:e1004490.

65. Hindi SM, Mishra V, Bhatnagar S, et al. Regulatory circuitry of TWEAK-Fn14 system and PGC-1 $\alpha$ in skeletal muscle atrophy program. FASEB J 2014;28:1398-411.

66. Liang J, Yang Y, Zhu X, et al. Down-expression of PGC1alpha partially mediated by JNK/c-Jun through binding to CRE site during apoptotic procedure in cerebellar granule neurons. J Neurosci Res 2010;88:1918-25.

67. Liu J, Chang F, Li F, et al. Palmitate promotes autophagy and apoptosis through ROS-dependent JNK and p38 MAPK. Biochem Biophys Res Commun 2015;463:262-7.

68. Ma CL, Qiao S, Li YC, et al. TGF- $\beta 1$ promotes human hepatic carcinoma HepG2 cells invasion by upregulating autophagy. Eur Rev Med Pharmacol Sci 2017;21:2604-10.

69. Yadav H, Quijano C, Kamaraju AK, et al. Protection from obesity and diabetes by blockade of TGF- $\beta / \mathrm{Smad} 3$ signaling. Cell Metab 2011;14:67-79.

70. Huang XZ, Wen D, Zhang $M$, et al. Sirt1 activation ameliorates renal fibrosis by inhibiting the TGF- $\beta / \mathrm{Smad} 3$ pathway. J Cell Biochem 2014;115:996-1005.

Cite this article as: Ma W, Zhang $\mathrm{R}$, Huang Z, Zhang Q, Xie X, Yang X, Zhang Q, Liu H, Ding F, Zhu J, Sun H. PQQ ameliorates skeletal muscle atrophy, mitophagy and fiber type transition induced by denervation via inhibition of the inflammatory signaling pathways. Ann Transl Med 2019;7(18):440. doi: 10.21037/atm.2019.08.101 